\title{
Urethral Villous Adenoma
}

National Cancer Institute

\section{Source}

National Cancer Institute. Urethral Villous Adenoma. NCI Thesaurus. Code C39872.

An epithelial neoplasm of the urethra, which is morphologically characterized by the presence of a villous architectural pattern. 